Paidéia, 2005, 15(32), 355-366

\title{
PERCEPÇÃO DE APOIO SOCIAL NAADOLESCÊNCIA: ANÁLISE FATORIAL CONFIRMATÓRIA DA ESCALA SOCIAL SUPPORT APPRAISALS ${ }^{1}$
}

\author{
Cristina Antunes ${ }^{2}$ \\ Anne Marie Fontaine \\ Universidade do Porto - Portugal
}

\begin{abstract}
Resumo: Durante a adolescência, a percepção de apoio social parece estar relacionada com o ajustamento psicossocial do adolescente e com o processo de desenvolvimento da sua auto-estima e da sua identidade. Este estudo aprecia algumas das qualidades psicométricas de um instrumento construído por Vaux para avaliar a percepção dos adolescentes do apoio emocional recebido da família, dos amigos e dos professores, o Social Support Appraisal (SSA). A sua consistência interna em estudantes portugueses do $7^{\circ}$ ao $12^{\circ}$ ano de escolaridade é satisfatória e melhora com a progressão na escolaridade. A sua estrutura, evidenciada graças ao recurso à técnica de modelação de estruturas covariantes (análise fatorial confirmatória, com o programa EQS, Bentler) revelou-se multidimensional e hierárquica, formada por um fator de segunda ordem e por três fatores de primeira ordem, que diferenciam a percepção de apoio social dos amigos, dos professores e da família.
\end{abstract}

Palavras-chave: Adolescência; apoio social; análise fatorial confirmatória.

\section{SOCIAL SUPPORT APPRAISALS DURING ADOLESCENCE: A CONFIRMATORY FACTOR ANALYSIS OF THE SOCIAL SUPPORT APPRAISALS SCALE.}

\begin{abstract}
Social support has been studied as an important variable, related to several aspects of Human behaviour and psychological well-being. During adolescence, the perception of support from the most important network resources - such as family, peers and teachers - seems to be related to the children's psychosocial adjustment, self-esteem and identity development. This study aimed to observe the psychometric qualities of an instrument developed by Vaux et al (1986) to assess the adolescents' perception of emotional support from family, peers and teachers. The "Social Support Appraisals" scale (SSA) was adapted to Portuguese students between $7^{\text {th }}$ and $12^{\text {th }}$ grades and it was analyzed in its reliability and factorial validity, using confirmatory factor analysis (CFA). Reliability revealed to be better in older students and CFA revealed a hierarchical structure, with a second order factor (emotional support) and three first order factors - emotional support from teachers, peers and family.
\end{abstract}

Key words: Adolescence; social support; factorial analysis.

Introdução: A conceptualização do apoio social refere-se a funções desempenhadas por grupo para um indivíduo, em determinadas situações da sua vida, e que podem ser familiares, amigos, vizinhos e outros, chamando-se ao conjunto por elas formado rede de relações sociais. A maioria dos estudos desenvolvidos nesta perspectiva privilegia, na análise do apoio social, a percepção que os indivíduos têm sobre

\footnotetext{
${ }^{1}$ Artigo recebido em 19/09/2005, aceito para publicação em 02/12/2005. ${ }^{2}$ Endereço para correspondência: Anne Marie Fontaine, Universidade do Porto, Rua do Campo Alegre, 1021/1055, Porto, Portugal, CEP: 4169-004, E-mail: fontaine@fpce.up.pt
}

si, que representa a crença generalizada por eles desenvolvida de que são estimados, que os outros se interessam por eles, que estão disponíveis quando precisam e satisfeitos com as relações que têm (Heller, Swindle \& Dusenbury, 1986; Vaux, 1988).

Um grande número de estudos enfatiza a importância do apoio social de outros significativos durante o desenvolvimento na adolescência, na relação deste constructo com o bem-estar físico e psicológico (Helsen, Vollebergh \& Meeus, 2000; Larose \& Bernier, 2001; Ystgaard, Tambs \& Dalgard, 1999), 


\section{Cristina Antunes}

com o autoconceito e a auto-estima (Antunes \& Fontaine, 1996; 2000; Harter, Waters \& Whitesell, 1998), assim como a sua relação com a motivação para o sucesso ou realização escolar (Wentzel, 1998).

Na maioria das pesquisas, investiga-se também a variação do impacto do apoio das principais redes sociais (pais, família, professores e amigos) ao longo da adolescência, que representa um período da vida que se caracteriza pela expansão das redes e modificação da influência de cada uma. Assim, o apoio social dos vários grupos de socialização pode variar com o tempo, pois nesse período ocorrem mudanças nas relações com os outros, nomeadamente pais e pares, e sua influência é crucial no desenvolvimento do adolescente, devido à estreita interdependência que se estabelece entre ele e o grupo, na qual parece se destacar a importância do apoio emocional, lealdade, compreensão e intimidade.

A amizade na adolescência tem sido conceitualizada como uma relação em que existe apoio e intimidade mútua (Youniss \& Smollar, 1989), e do início para seu final, o dos amigos parece se tornar mais relevante que o dos pais (Epstein,1989; Sprinthall \& Collins, 1988; Scholte, Lieshout \& Aken, 2001). Contudo, é necessário clarificar de que tipo de apoio se trata e há autores sugerindo que durante a adolescência, as relações com os outros significativos devem ser contrabalançadas, para que ele possa lidar eficazmente com as tarefas desenvolvimentais do período (Colarossi \& Eccles, 2003; DuBois, Burk-Braxton, Swenson, Tevendale, Lockerd \& Moran, 2002).

Antunes (1994) observou, num estudo transversal com alunos do sexto ao décimo ano, que a percepção de apoio emocional da família era superior à dos pares em todos os anos de escolaridade. Já Meeus (1999) revelou que a influência dos pares na adolescência está limitada a áreas como o lazer, enquanto a dos pais é mais importante frente às relações pessoais e escola; assim, embora ao longo da adolescência o grupo de pares se torne essencial na socialização e processo de construção de identidade, a família o é para o bem-estar; então, o apoio social dos pais parece ter importância semelhante à do grupo de pares. (Meeus, Helsen \& Vollebergh,1996)

Também os professores constituem um grupo de adultos cujo apoio seria relevante para os adolescentes, sobretudo quanto ao seu autoconceito escolar
(Antunes \& Fontaine,1996, 2000; Pekrun, 1990) e mesmo auto-estima. (Colarossi \& Eccles, 2003). Eles podem ser veículo de promoção e ampliação dos direitos psicossociais dos jovens no seio da família e ter ainda papel na construção da sua identidade (Veiga, 1999). DuBois e cols. (2002), Harter e cols. (1998) sugerem que o apoio social dos professores poderia reduzir o efeito de acontecimentos negativos no início da adolescência. Em suma, se o adolescente precisa de mais intimidade com os pares, também necessita do apoio da família e dos professores para lidar com as exigências e expectativas da aprendizagem escolar (Furman \& Burmester; 1985). Perceber, a influência dos grupos sociais mais significativos é importante, porque contribuem, juntamente com outros mecanismos desenvolvimentais, para a construção do autoconceito e da identidade na adolescência.

\section{Avaliação do Apoio Social}

Os problemas na investigação sobre o apoio social colocam-se quer no nível do conceito da variável, quer da sua operacionalização, com implicações para as qualidades psicométricas dos instrumentos utilizados. Numa perspectiva transacional e ecológica (Vaux, 1992) refere-se a um processo dinâmico e complexo, envolvendo transações entre os indivíduos e as suas redes de apoio, em que as pessoas regulam seus recursos sociais de modo a atingir seus objetivos. O tipo de apoio dado, além de ser uma variável dependente do contexto, envolve vários comportamentos e serve a diversas funções.

Na avaliação, os instrumentos utilizados devem ir ao encontro dos objetivos preconizados. No caso da investigação epidemiológica, que se preocupa com a incidência de certos fenômenos na população global, os instrumentos de avaliação que focam a amplitude dos recursos de apoio social ou as percepções gerais sobre ele são os mais usuais. Estudos relativos a fatores de stress em situações específicas (estudar o papel do apoio social numa relação marital ou de trabalho, para deixar de fumar ou beber), usam instrumentos que especificam o tipo de apoio percebido ou recebido (emocional, prático, financeiro), as fontes ou recursos dele, relacionados com o fator de stress frente ao qual é providenciado. No caso em que a avaliação tem objetivos clínicos, os instrumentos devem procurar verificar, se o cliente tem uma orientação negativa 
face à procura de apoio, uma percepção negativa dele ou se possui rede de apoio que facilitará a gestão de transições na sua vida. Nestes casos, recorre-se principalmente à entrevista ou administra-se questionário à pessoa e membros de sua rede, sempre focalizados nos aspectos do apoio social que importa avaliar, antes, durante e depois da intervenção.

Em Portugal, existem diversas escalas de avaliação do apoio social, para adultos, para crianças e adolescentes, algumas originais, outras adaptadas de escalas estrangeiras. Nas de apoio social destinadas a adultos, destacam-se: a "Social Support Questionnaire" (SSQ6) de Sarason, Shearin e Pierce (1987), adaptada por Pinheiro e Ferreira (2002), com qualidades psicométricas testadas junto a estudantes universitários; a "Escala de Apoio Social Instrumental e Expressivo” (IESSS), adaptada a partir da "Instrumental and Expressive Social Support Scale" de Lin, Dean e Ensel (Sousa Faria, 1999).

Uma das escalas para crianças ou adolescentes, a "Social Support Scale for Children" (SSSC) de Harter, adaptada por Almeida, Reis e Norton (1999), avalia o apoio que cada criança sente que recebe das pessoas significativas do seu meio: pais, colegas, amigos íntimos e professores. Embora os valores de sua consistência interna sejam razoáveis, por ser um estudo exploratório, ele não é uma adaptação válida da escala para a população juvenil portuguesa, em geral, tendo sido usada numa amostra de trinta crianças e adolescentes sobreviventes de leucemia, com idades entre os oito e os dezessete anos.

Além desta, uma "Escala de Percepção da Relação com a Família” (EPRF) foi construída por Peixoto (1999) com o objectivo de avaliar a relação dos adolescentes com os seus pais, em vários domínios, como a aceitação, o suporte afetivo, as expectativas, a autonomia, o envolvimento nas tarefas escolares, reforço e punição. Esta escala revelou boa consistência interna, embora melhor para os adolescentes mais velhos.

A escala "Social Support Appraisals" (SSA), objeto do estudo que aqui se apresenta, é contudo de âmbito mais amplo, na medida em que não se limita à família mas avalia também a percepção do apoio social recebido de outros grupos sociais. Foi traduzida por Antunes (1994) da original americana desenvolvida por Vaux e cols (1986). Baseia-se na perspectiva de Cobb
(1976) de apoio social, ou seja, na crença de que se é amado, respeitado, estimado e afiliado a grupos. Estas crenças são uma percepção subjetiva fornecida pela ocorrência de interações confortantes e apoiantes, relacionadas com o tamanho real das redes de apoio, sua composição, a proximidade das relações (Vaux e Harrison, 1985). Vaux e cols. (1986) demonstraram a validade e fidedignidade da escala SSA com cinco amostras de estudantes e cinco de pessoas da comunidade. A escala total (SSA), a sub-escala SSA-fam (apoio social da família) e a sub-escala SSA-am (apoio social dos amigos) revelaram boa consistência interna nos dois tipos de amostra (para os estudantes, o coeficiente alpha de Cronbach foi de $\alpha=.90, \alpha=.80$ e $\alpha=.84$, para SSA, SSA-fam e SSA-am, respectivamente, e para a comunidade foi de $\alpha=.90, \alpha=.81$ e $\alpha=.71$ ). As escalas revelaram ainda estabilidade num intervalo de seis semanas $(\mathrm{r}=.80$ e .71 , para as sub-escalas SSAfam e SSA-am) e uma sobreposição moderada ( $\mathrm{r}=.51$ e $r=.52$, para as amostras de estudantes e da comunidade).

A validade convergente foi demonstrada através das associações com sete outras medidas de percepção, com variáveis de recursos da rede de apoio (tais como proporção de amigos íntimos, tamanho da rede de apoio emocional), medidas de bem-estar psicológico (como depressão, solidão, satisfação, felicidade, otimismo) e fatores de personalidade (como orientação positiva face à rede de apoio, autonomia, afiliação).

A versão portuguesa do SSA (Antunes, 1994) divide-se em quatro sub-escalas: além da percepção de apoio das outras pessoas, avalia a referente à família, amigos, professores (esta última escala não existia na versão americana original), com oito, oito, sete e sete itens, respectivamente. Os itens apresentam afirmações frente as quais o adolescente deve exprimir o seu grau de concordância. $\mathrm{O}$ formato de resposta corresponde a uma escala de Lickert com alternativas que variam de "concordo totalmente" a “discordo totalmente”; para alunos até o oitavo ano, a escala tem quatro níveis e para os do nono ano em diante, tem seis níveis. A pontuação em cada item cresce no sentido de uma percepção positiva, mesmo para os formulados negativamente. O total de pontuação, em cada sub-escala ou dimensão, corresponde assim à percepção positiva de apoio social, obtida so- 
mando os pontos dos itens que as compõem. A multidimensionalidade do instrumento e a consistência interna das subescalas foram confirmadas numa amostra de 364 adolescentes Portugueses, do quinto, sexto e nono ano de escolaridade. A estrutura fatorial do SSA, mediante a técnica de análise em componentes principais, confirmou as quatro dimensões teóricas. Foram observados valores de alpha de Cronbach satisfatórios $\alpha=.79$ para a percepção de apoio social dos amigos, $\alpha=.80$ da família e $\alpha=.79$ dos professores (Antunes \& Fontaine, 1995).

\section{Objetivos}

O estudo aqui apresentado pretende, com uma amostra mais heterogênea que a anterior, confirmar as qualidades psicométricas da Social Support Appraisals (SSA), nomeadamente seu caráter multidimensional, diferenciando a percepção de apoio social dos adolescentes em relação à família, amigos e professores e a consistência das sub-escalas ${ }^{3}$.

\section{Método}

O estudo das qualidades psicométricas desta escala foi realizado numa amostra de 1963 adolescentes de ambos os sexos, do $7^{\circ}$ ao $12^{\circ}$ ano de escolaridade (início do ano letivo de 2001/2002), provenientes da cidade do Porto (litoral) e de três concelhos de Trás-os-Montes e Alto Douro (interior), sendo a escala administrada de forma coletiva em sala de aula, juntamente com outras de avaliação psicológica, apresentadas de forma contrabalançada por psicólogas com experiência de investigação. O tempo médio de resposta à escala SSA foi de dez minutos.

\footnotetext{
3 A dimensão percepção de apoio social dos outros em geral, presente tanto na versão americana como na sua adaptação (Antunes, 1994), não foi incluída neste estudo. Considera-se que, tanto em termos de investigação, na relação com outros construtos psicológicos, quanto em contextos clínicos, a percepção do apoio das redes específicas é mais relevante. Além disso, a percepção dos adolescentes relativamente ao apoio social emocional, ou seja, a estima, o respeito e a compreensão que sentem que recebem da família, amigos e professores pode dar uma ideia do apoio social em geral, sendo estes os grupos de maior relevo na rede de relações sociais na adolescência.
}

Análise das qualidades psicométricas da SSA

A consistência interna (coeficiente alpha de Cronbach) em cada dimensão (sub-escalas) do SSA permite retirar os itens menos consistentes para depois testar a estrutura multidimensional da escala, recorrendo à análise fatorial confirmatória (AFC) usando o programa EQS (Bentler, 1995).

\section{Percepção de Apoio Social dos Professores}

A sub-escala percepção de apoio social dos professores apresenta, no geral, mas sobretudo para alunos do $7^{\circ}$ e $8^{\circ}$ anos, dois itens problemáticos: 14 e 21, que estão formulados na negativa (14: “Na generalidade, não posso contar com os meus professores para me darem apoio" e 21: "Não me sinto muito chegado aos meus professores"), o que pode explicar a baixa correlação observada entre eles e o total da dimensão, nos $7^{\circ}$ e $8^{\circ}$ anos. A eliminação do item 21 não faria, no geral, subir o valor do alpha, mas a do $14 \mathrm{sim}$; o valor de alpha vai de $\alpha=.72$ até $\alpha=.77$ no $7^{\circ}$ ano e de $\alpha=.72$ até $\alpha=.76$ no $8^{\circ}$ ano. Resultados semelhantes foram observados nas subamostras constituídas por meninas e rapazes, do $7^{\circ} \mathrm{e}$ $8^{\circ}$ ano. Para elas o valor de alpha iria de $\alpha=.75$ a $\alpha=.79$ se o item 14 fosse eliminado e de $\alpha=.69$ até $\alpha=.73$ no caso dos rapazes (Quadro II). 
Quadro II - Consistência interna por ano de escolaridade e sexo na sub-escala percepção de apoio social dos professores (alpha de Cronbach).

\begin{tabular}{|c|c|c|c|c|}
\hline $\begin{array}{c}\text { Ano de } \\
\text { escolaridade }\end{array}$ & $\begin{array}{c}\mathrm{N}^{\circ} \text { de } \\
\text { itens }\end{array}$ & $\mathrm{n}$ & alpha & $\begin{array}{c}\text { Valor de alpha se } \\
\text { item 14 for apagado }\end{array}$ \\
\hline $7^{\circ}$ & 7 & 257 & .72 & .77 \\
$8^{\circ}$ & 7 & 293 & .72 & .76 \\
$9^{\circ}$ & 7 & 351 & .77 & .79 \\
$10^{\circ}$ & 7 & 478 & .82 & Não se altera \\
$11^{\circ}$ & 7 & 342 & .85 & Não se altera \\
$12^{\circ}$ & 7 & 242 & .85 & Não se altera \\
\hline Sexo & & & & .73 \\
\hline Masc. $7^{\circ} / 8^{\circ}$ & 7 & 262 & .69 & .79 \\
Fem. $7^{\circ} / 8^{\circ}$ & 7 & 288 & .75 & Não se altera \\
Masc. $9^{\circ} / 12^{\circ}$ & 7 & 559 & .80 & Não se altera \\
Fem. $9^{\circ} / 12^{\circ}$ & 7 & 854 & .84 \\
\hline
\end{tabular}

Nos $9^{\circ}, 10^{\circ}, 11^{\circ}$ e $12^{\circ}$ anos, os valores de alpha, caso o item 14 fosse eliminado, não subiriam; este item foi também o que apresentou, sobretudo nos grupos de $9^{\circ}$ e $10^{\circ}$ anos e das meninas, as correlações mais baixas com o total da dimensão. Os resultados obtidos a partir da análise das correlações item-total e da observação dos valores de alpha se os itens 14 e 21 fossem eliminados, sugerem que a consistência interna da sub-escala "Percepção de apoio social dos professores" seria mais robusta se suprimidos os referidos itens.

\section{Percepção de Apoio Social da Família}

A sub-escala que avalia a percepção de apoio social da família apresentou valores alpha situados entre .77 e .90 , e que poderiam subir, em alguns casos, se o item 22 ("Não me sinto muito ligado à minha família") ou o 17 ("Não posso contar com a minha família para me dar apoio”) fossem suprimidos. Estes itens fazem baixar o valor de alpha nos alunos do $7^{\circ}$, $8^{\circ}$ e $9^{\circ}$ anos de escolaridade e nos rapazes do $7^{\circ}$ e $8^{\circ}$ ano. (Quadro III).

\section{Percepção de Apoio Social dos Amigos}

Relativamente à sub-escala que avalia a percepção do apoio social dos amigos, verificou-se que o item 8 ("Os meus amigos não se preocupam nada comigo”), formulado negativamente, é problemático, apresentando os valores mais baixos nas correlações item-total e, se fosse eliminado, levaria a melhoria na consistência interna desta sub-escala, sobretudo nos alunos do $7^{\circ}$ e $8^{\circ}$ ano (Quadro IV).
Quadro III - Consistência interna por ano de escolaridade e sexo na sub-escala percepção de apoio social da família (alpha de Cronbach).

\begin{tabular}{|c|c|c|c|c|}
\hline $\begin{array}{c}\text { Ano de } \\
\text { escolaridade }\end{array}$ & $\begin{array}{l}\mathrm{N}^{\circ} \text { de } \\
\text { itens }\end{array}$ & $\mathrm{n}$ & alpha & $\begin{array}{l}\text { Valor de alpha se } \\
\text { item for apagado }\end{array}$ \\
\hline $7^{\circ}$ & 8 & 257 & .77 & $\begin{array}{c}.79 \text { (se item } 22 \\
\text { apagado) }\end{array}$ \\
\hline $8^{\circ}$ & 8 & 293 & .84 & $\begin{array}{c}.85 \text { (se item } 22 \\
\text { apagado) }\end{array}$ \\
\hline $9^{\circ}$ & 8 & 351 & .84 & $\begin{array}{c}.86 \text { (se item } 17 \\
\text { apagado) }\end{array}$ \\
\hline $10^{\circ}$ & 8 & 478 & .89 & Não se altera \\
\hline $11^{\circ}$ & 8 & 342 & .92 & Não se altera \\
\hline $12^{\circ}$ & 8 & 242 & .90 & Não se altera \\
\hline \multicolumn{5}{|l|}{ Sexo } \\
\hline Masc. $7^{0} / 8^{\circ}$ & 8 & 262 & .79 & $\begin{array}{c}.81 \text { (se item } 22 \text { for } \\
\text { apagado) }\end{array}$ \\
\hline Fem. $7^{\circ} / 8^{\circ}$ & 8 & 288 & .82 & Não se altera \\
\hline $\begin{array}{l}\text { Masc. } 9^{\circ} \\
\qquad / 12^{\circ}\end{array}$ & 8 & 559 & .87 & Não se altera \\
\hline Fem. $9^{\circ} / 12^{\circ}$ & 8 & 854 & .90 & Não se altera \\
\hline
\end{tabular}

Quadro IV - Consistência interna por ano de escolaridade e sexo na sub-escala percepção de apoio social dos amigos (alpha de Cronbach).

\begin{tabular}{|c|c|c|c|c|}
\hline $\begin{array}{c}\text { Ano de } \\
\text { escolaridade }\end{array}$ & $\begin{array}{l}\mathrm{N}^{\circ} \text { de } \\
\text { itens }\end{array}$ & $\mathrm{n}$ & alpha & $\begin{array}{l}\text { Valor de alpha se } \\
\text { item for apagado }\end{array}$ \\
\hline $7^{\circ}$ & 7 & 257 & .81 & $\begin{array}{c}.84 \text { (se item } 8 \\
\text { apagado) }\end{array}$ \\
\hline $8^{\circ}$ & 7 & 293 & .86 & $\begin{array}{c}.87 \text { (se item } 8 \\
\text { apagado) }\end{array}$ \\
\hline $9^{\circ}$ & 7 & 351 & .83 & $\begin{array}{c}.84 \text { (se item } 8 \\
\text { apagado) }\end{array}$ \\
\hline $10^{\circ}$ & 7 & 478 & .83 & $\begin{array}{c}.84 \text { (se item } 8 \\
\text { apagado) }\end{array}$ \\
\hline $11^{\circ}$ & 7 & 342 & .90 & Não se altera \\
\hline $12^{\circ}$ & 7 & 242 & 88 & Não se altera \\
\hline \multicolumn{5}{|l|}{ Sexo } \\
\hline Masc. $7^{0} / 8^{0}$ & 7 & 262 & .83 & $\begin{array}{c}.85 \text { (se item } 8 \\
\text { apagado) }\end{array}$ \\
\hline Fem. $7^{\circ} / 8^{\circ}$ & 7 & 288 & .84 & .86 (se item 8 for \\
\hline Masc. $9^{\circ} / 12^{\circ}$ & 7 & 559 & .86 & Não se altera \\
\hline Fem. $9^{\circ} / 12^{\circ}$ & 7 & 854 & .86 & Não se altera \\
\hline
\end{tabular}

Em suma, pode-se dizer que a consistência interna das três sub-escalas da SSA é razoável ou mesma boa, sendo melhor nos participantes mais velhos, sobretudo a partir do $10^{\circ}$ ano de escolaridade e, nas sub-amostras das meninas. Estes resultados podem espelhar as diferentes fases de maturação dos sujeitos, que se traduz na progressiva melhoria da compreensão do significado semântico dos itens, e 
da capacidade de análise e síntese pessoal face às características invocadas. Para os mais novos, $7^{\circ} \mathrm{e}$ $8^{\circ}$ anos, os itens formulados na negativa parecem diminuir a consistência interna da escala. Embora a formulação na negativa tenha como propósito impedir o enviesamento da resposta numa determinada opção, nestas idades é provavelmente difícil compreender o significado afirmativo da dupla negação, uma vez que a resposta tipo Likert inclui as alternativas "discordo" e "discordo totalmente". A decisão foi eliminar estes itens das escalas.

A consistência interna mais baixa na dimensão “apoio social dos professores” do que nas outras duas dimensões - da família e dos amigos - poderá advir do fato de o enunciado dos itens se referir a "professores", de uma forma generalizada. Não se sabe em quais professores os alunos pensam quando respondem ao questionário: se naqueles com quem tiveram/ têm um relacionamento mais positivo ou nos que mantém relacionamento mais negativo? Além disso, os professores são diferentes uns dos outros e mudam todos os anos, ao invés da família, que permanece, assim como, para a maioria, os amigos.

\section{Análise Fatorial Confirmatória}

A análise fatorial confirmatória (AFC) é uma técnica multivariada que tem como requisitos básicos os mesmos de todas as multivariadas - linearidade, normalidade, ausência de multicolinearidade e homocedasticidade das variâncias residuais (Giles, 2002). Embora o racional teórico subjacente à técnica de modelação de estruturas covariantes, na qual a análise fatorial confirmatória se inclui, tenha sido inicialmente desenvolvida pelos investigadores suecos, nos anos 70 e 80 do séc. XX (Jöreskog \& Sörbon, 1983), existem hoje vários programas que permitem a modelação de estruturas covariantes, como o AMOS (Arbuckle, 1997) e o EQS (Bentler, 1995; Bentler e Weeks, 1980).

\section{Características dos modelos de equações estruturais}

Na modelação de equações estruturais, técnica onde se inclui a AFC, os modelos têm variáveis observadas e latentes, o que constitui uma das vantagens deste tipo de análise. Efetivamente, ela permite ao investigador testar a plausibilidade de um modelo teórico, baseado na assumção de que certas variáveis inferidas (a quase generalidade das estudadas em psicologia), podem ser estimadas a partir das observadas (itens de uma escala). Além disso, é também uma vantagem desta técnica a possibilidade de assumir nos modelos os erros associados à observação de variáveis e às latentes, que não sendo verdadeiramente "erros", são designados por efeitos aleatórios ou perturbações.

Uma outra vantagem desta técnica, em relação às multivariadas tradicionais de análise fatorial, é a de permitir responder à questão: será que todos os itens da escala estimam do mesmo modo, com a mesma magnitude, o fator em questão? Se a resposta a esta questão for afirmativa, estará preenchida a condição subjacente ao cálculo do valor da escala para cada sujeito, que normalmente se faz por simples adição do obtido por ele em cada item. Se, pelo contrário, a variância do fator explicada por item for substancialmente diferente, então, justificar-se-ia levar em consideração e ponderar cada item em função da percentagem de variância explicada pelo fator subjacente à escala.

A questão fundamental que se coloca ao realizar AFC é saber se os padrões de covariância entre as variáveis observadas são “causados” pela variância nos fatores. O número de fatores, assim como a relação entre eles, são decididos pelo investigador, com base em motivos de ordem substantiva ou teórica. $\mathrm{O}$ programa EQS, procedimento de máxima verosimilhança, faz uma estimativa dos parâmetros, refinando-os gradualmente, até não poderem "ficar melhor”. Em cada estádio, compara a matriz de covariâncias observadas com a esperada/estimada e tenta encontrar os valores que permitam aumentar a semelhança entre as duas (Maia, 1996; Schumacker \& Lomax, 1996).

\section{Critérios de ajustamento dos modelos}

O modelo final é normalmente escolhido com base em critérios subjectivos (formulações teóricas), mas em seu auxílio, o programa EQS (tal como todos os de modelação de estruturas covariantes) fornece alguns índices que permitem ter uma idéia da adequação do modelo. Esse ajustamento pode ser apreciado através dos chamados índices global e local, que mais não são do que os valores de saturação dos 
fatores ou variáveis latentes, os valores únicos de cada item ou variâncias-erro $\mathrm{R}^{2}$, que alteram as latentes.

Os índices de ajustamento global mais referenciados na literatura são o CFI ("comparative fit index"), o GFI ("goodness of fit index”), RMR ("root mean square residual") e RMSEA ("root mean square error of approximation").

O CFI, desenvolvido por Bentler (1990) e semelhante ao RNI ("relative noncentrality index"), de MacDonald e Marsh (1990), compara o modelo estimado com um completamente independente, no qual não existe relação entre as variáveis, com base no procedimento de máxima verosimilhança. Este índice varia entre 0 e 1 e os valores superiores a 0.90 indicam um ajustamento aceitável.

O GFI, introduzido por Jöreskog e Sörbon (1983) é um índice que compara a matriz de covariância da amostra utilizada com a da estimada para a população, isto é, que parâmetros poderiam ser esperados de replicações do estudo em diferentes amostras. Tal como o anterior, o seu valor está compreendido entre 0 e 1 e os acima de 0.90 indicam um ajustamento aceitável.

O RMR (Arbuckle, 1997) representa a diferença entre as matrizes de covariância da amostra estudada e as da população. Portanto, quanto menor o seu valor, menor é a diferença e mais aceitável o modelo; os valores variam entre 0 (nenhuma diferença) e 1 (diferença máxima) e os inferiores a 0,050 indicam um ajustamento aceitável. Posteriormente, Browne e Cudeck (1993), a partir do RMR, introduziram o RMSEA, que é um índice que não penaliza a complexidade do modelo e tende mesmo a favorecer os modelos mais complexos. Este índice dá a idéia da diferença existente entre a matriz de variâncias e covariâncias da amostra e do modelo obtido, partindo do pressuposto que o ele é adequado. Neste sentido, quanto mais próximo de 0 for o seu valor, maior a proximidade entre a matriz de variâncias e covariâncias estimada e a dos dados e mais "segurança” para não rejeitar o modelo obtido. Valores inferiores a 0.08 indicam ajustamento aceitável (Arbuckle, 1997), quando o intervalo de confiança é de $90 \%(\mathrm{p}<.10)$.

Finalmente, utiliza-se ainda o $X^{2}$, não como um teste estatístico, mas como um índice de ajustamento do modelo, que exprime, juntamente com o número de graus de liberdade, a distância entre o modelo hipotético especificado e o sugerido pelos dados. O ideal é obter um $X^{2}$ mais baixo possível e não significativo, mas como esta estatística é sensível, pela sua fórmula, ao tamanho da amostra, utiliza-se muitas vezes o valor obtido na divisão do $X^{2}$ pelo número de graus de liberdade (rácio $X^{2} / \mathrm{gl}$ ), considerando um ajustamento aceitável do modelo quando esse valor é inferior a 5 (Giles, 2002), embora isto não seja pacífico entre os investigadores. Marsh e Hocevar (1985) sugeriram que um valor entre 2 e 5 indicaria um ajustamento aceitável, enquanto para Byrne (1989) os superiores a 2 não deveriam ser aceitos. $O$ valor do $X^{2}$ também é utilizado na comparação entre modelos testados de complexidade crescente, para decidir qual escolher. Normalmente, subtraindo os valores do $X^{2}$ e do número de graus de liberdade, verifica-se se a diferença é significativa, preferindo-se, neste caso, o modelo mais complexo, com menor valor de $X^{2}$.

\section{Modelos hipotéticos da estrutura fatorial da escala SSA}

Uma vez que o formato de resposta da escala é diferente para alunos até o oitavo ano (quatro alternativas de resposta tipo Lickert, variando entre "concordo totalmente" e "discordo totalmente") e do nono ano em diante (seis alternativas de resposta tipo Lickert, variando entre "concordo totalmente" e "discordo totalmente"), optou-se por apresentar os estudos da AFC em duas sub-amostras: alunos do $7^{\circ}$ e $8^{\circ}$ ano $(n=550)$ e do $9^{\circ}$ ao $12^{\circ}$ ano $(n=1413)$. Os itens com formulação negativa, por terem suscitado problemas na consistência interna, não foram incluídos na AFC.

Em cada sub-amostra foram testados três modelos de estrutura fatorial da SSA. No primeiro sugerese que a percepção de apoio social é um constructo de segunda ordem, cuja variância é explicada por três fatores, que correspondem às três dimensões da escala percepção de apoio social da família (SSA-fam), dos amigos (SSA-am) e dos professores (SSA-prof), que constituem fatores de primeira ordem, cuja variância é explicada pelos respectivos itens (oito, sete e sete itens, respectivamente), de forma igual, com variância-erro também igual, dentro de cada fator. No segundo modelo sugere-se que a variância de cada um dos três fatores de primeira ordem é explicada com igual magnitude 


\section{Cristina Antunes}

pelos respectivos itens, cuja variância-erro, contudo, não é a mesma em todos os itens (tau-equivalente). Num terceiro modelo, testa-se a possibilidade de todos os itens explicarem, com magnitude diferente, o respectivo fator de primeira ordem, assim como de serem diversas as variâncias-erro associadas a cada item (congenérico). Estes três modelos são de complexidade crescente, uma vez que os parâmetros a estimar vão aumentando de um para outro. $\mathrm{O}$ fato de se testarem modelos de configuração hierárquica, isto é, que supõem um fator de segunda ordem, explica-se pela existência da correlação entre os três fatores. Na verdade, quando dois ou mais de primeira ordem estão bastante correlacionados, espera-se que exista um fator de ordem hierarquicamente superior responsável pela variância partilhada por eles. Em termos substantivos ou teóricos, faz sentido pensar na percepção de apoio social em geral, como fator de ordem hierarquicamente superior, cuja variância é explicada pela percepção do apoio social emocional das pessoas mais significativas da rede dos adolescentes, família, amigos e professores. Na fig. 1 apresenta-se a especificação pictográfica do modelo geral de AFC a testar.

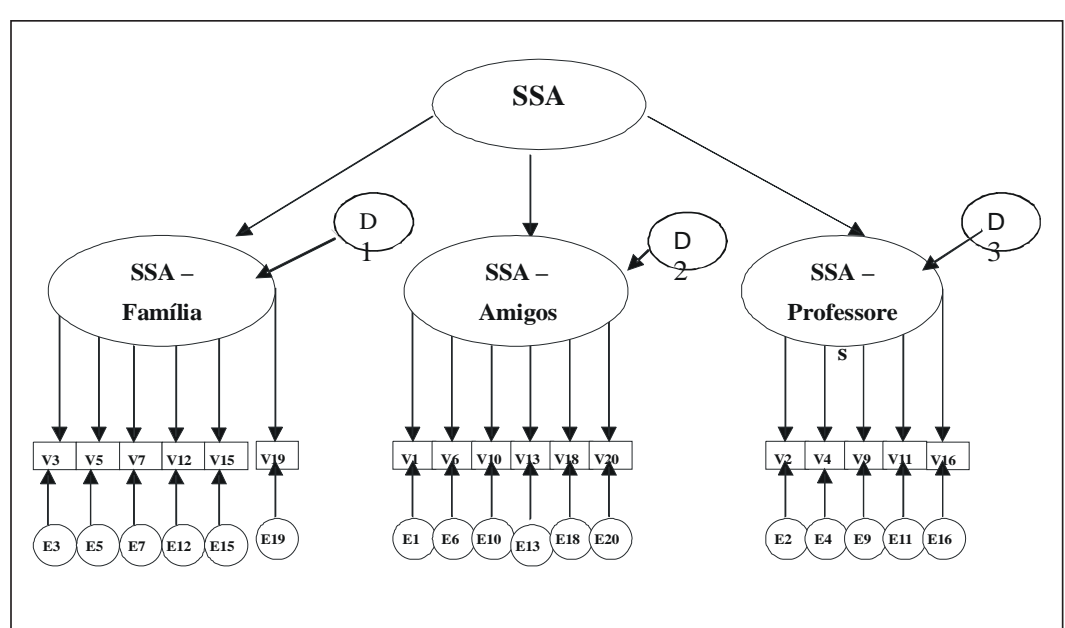

No quadro decisional (V), pode-se observar os índices de ajustamento global obtidos para cada um dos três modelos testados, na sub-amostra do $7^{\circ}$ e $8^{\circ}$ anos de escolaridade. Verifica-se que o modelo paralelo não obteve um ajustamento global tão bom como os tau-equivalente e congenérico. Entre estes dois, optou-se pelo tau-equivalente como um que explica de forma suficiente os dados. Embora apenas o paralelo permitisse assumir que todos os itens explicam a mesma percentagem de variância do fator correspondente, com o fim de somar a sua pontuação na obtenção de um valor total da dimensão que se avalia, considerou-se não ser ilegítimo manter essa assumpção no caso do tau-equivalente, visto que as saturações são iguais para todos os itens, variando unicamente a variância-erro associada a cada item. $\mathrm{O}$ valor alpha obtido para o tau-equivalente global foi de $\alpha=.89$. O do índice rho (que supõe um “peso” diferente de cada item na explicação da variância do fator) foi de .92. A diferença mínima entre os dois legitima também a opção pelo tau-equivalente.

No quadro VI observam-se os índices de ajustamento local do modelo tauequivalente, ou seja, os valores da variância explicada por cada fator nos itens $\left(\mathrm{R}^{2}\right)$, a variância-erro associada aos itens e a saturação do fator geral ou de segunda ordem (SSA) em cada fator de primeira ordem (SSA-fam, SSA-am e SSA-prof).

Quadro V - Quadro decisional: Valores dos índices de ajustamento global de três modelos testados na AFC nas subamostras do $7^{\circ}$ e $8^{\circ}$ ano de escolaridade $(\mathrm{n}=550)$ e do $9^{\circ}$ ao $12^{\circ}$ ano $(\mathrm{n}=1413)$
Fig. 1: Especificação pictográfica do modelo de análise fatorial confirmatória da escala SSA .

Legenda: SSA - percepção de apoio social emocional total; SSA-Família = percepção de apoio social da família; SSAAmigos = percepção de apoio social dos amigos; SSAprofessores = percepção de apoio social dos professores; $\mathrm{V}$ = variáveis observadas (itens); $\mathrm{E}=$ variância-erro associada ao item; D1 a D3 = variância aleatória (“disturbance”) associada ao fator ou variável inferida.

\begin{tabular}{|c|c|c|c|c|c|c|c|c|}
\hline & \multicolumn{7}{|c|}{ Índices de ajustamento global } & \multicolumn{2}{|c|}{} \\
\hline $7^{\circ}$ e ${ }^{\circ}$ ano & CFI & GFI & RMR & RMSEA & $\mathrm{X}^{2}$ & Gl & Dif. $\mathrm{X}^{2}$ & Dif.G1 \\
\hline Independente & & & & & 4037.806 & 150 & & \\
Paralelo & .864 & .861 & .033 & $.084^{2}$ & 673.388 & 144 & 235.582 & 6 \\
Tau-equivalente & .933 & .920 & .029 & $.061^{2}$ & 384.346 & 130 & 289.042 & 14 \\
Congenerico & .943 & .928 & .021 & $.060^{2}$ & 332.804 & 116 & 51.542 & 14 \\
\hline $9^{\circ}$ ao $12^{\circ}$ ano & & & & & & & & \\
\hline Independente & & & & & 12389,56 & 150 & & \\
\hline Paralelo & .853 & .849 & .076 & $.096^{2}$ & 1939,45 & 144 & 10450,11 & 6 \\
\hline Tau-equivalente & .923 & .913 & .065 & $.072^{2}$ & 1051,56 & 130 & 887,89 & 14 \\
\hline
\end{tabular}

Dif. = diferença ; $\mathrm{X}^{2}=$ qui-quadrado;

$\mathrm{Gl}=$ graus de liberdade; ${ }^{2} \mathrm{p}<.10$ 
Quadro VI: Quadro dos índices de ajustamento local (valores padronizados) do modelo tau-equivalente, subamostra $9^{\circ}$ ao $12^{\circ}$ anos de escolaridade $(n=1413)$.

\begin{tabular}{|c|c|c|c|c|}
\hline Variáveis & fator & $\begin{array}{c}\text { Valor } \\
\text { de v }\end{array}$ & $\begin{array}{c}\text { Valor } \\
\text { de e }^{3}\end{array}$ & $\mathrm{R}^{2(*)}$ \\
\hline V1(item1) & SSA-am & .703 & .711 & .495 \\
\hline V6 (item6) & SSA-am & .681 & .733 & .463 \\
\hline V10 (item 10) & SSA-am & .702 & .713 & .492 \\
\hline V13 (item 13) & SSa-am & .724 & .690 & .525 \\
\hline V18 (item 18) & SSA-am & .745 & .667 & .555 \\
\hline V20 (item 20) & SSA-am & .748 & .664 & .559 \\
\hline V2(item 2) & SSA-prof & .720 & .694 & .518 \\
\hline V4 (item 4) & SSA-prof & .752 & .659 & .566 \\
\hline V9 (item 9) & SSA-prof & .677 & .736 & .459 \\
\hline V11 (item 11) & SSA-prof & .766 & .643 & .586 \\
\hline V16 (item 16) & SSA-prof & .724 & .690 & .524 \\
\hline V3 (item 3) & SSA-fam & .805 & .594 & .647 \\
\hline V5 (item 5) & SSA-fam & .837 & .547 & .701 \\
\hline V7 (item 7) & SSA-fam & .626 & .780 & .392 \\
\hline V12 (item 12) & SSA-fam & .845 & .535 & 713 \\
\hline V15 (item 15) & SSA-fam & .741 & .671 & .549 \\
\hline V19 (item 19) & SSA-fam & .831 & .556 & .691 \\
\hline SSA-am & Fator 1 & .688 & .726 & $.473^{4}$ \\
\hline SSA- Prof & Fator 2 & .678 & .735 & $.460^{4}$ \\
\hline SSA-fam & Fator 3 & .711 & .703 & $.506^{4}$ \\
\hline
\end{tabular}

Legenda: SSA-am = percepção de apoio social dos amigos; SSA-prof = percepção de apoio social dos professores; SSA-família= percepção de apoio social da família;

${ }^{1}$ loading do fator em cada variável observada; ${ }^{3}$ variância residual do item(variência-erro);(*)Variância do fator explicada por cada item/variável observada; ${ }^{4}$ Variância do erro associado às variáveis (fatores) latentes (“disturbance”). Nota: todos os valores apresentados se encontram padronizados ou estandardizados.

São também disponíveis no quadro $\mathrm{V}$ os índices de ajustamento global na sub-amostra do $9^{\circ}$ ao $12^{\circ}$ anos de escolaridade, na qual foram também testados os três modelos anteriormente especificados sem incluir os itens formulados na negativa.

Ao se analisarem os índices de ajustamento local dos modelos (Quadro VII), verifica-se que os mais ajustados, tal como na sub-amostra do $7^{\circ}$ e $8^{\circ}$ ano, são os modelos tau-equi e congenérico.

No modelo tau-equivalente, o $\mathrm{R}^{2}$ varia entre .39 e .71. No congenérico, estes valores de $\mathrm{R}^{2}$ oscilaram entre .35 e .69. Em face destes resultados, e porque foi também o modelo escolhido na sub-amostra do $7^{\circ}$ e $8^{\circ}$ anos de escolaridade, decidiu-se optar pelo modelo tau-equivalente, como solução fatorial da escala SSA, adequado aos dados da sub-amostra de alunos do $9^{\circ}$ ao $12^{\circ}$ ano.

QuadroVII: Índices de ajustamento local (valores estandardizados) modelo tau-equivalente, sub-amostra do $7^{\circ}$ e $8^{\circ}$ anos de escolaridade $(n=550)$.

\begin{tabular}{|c|c|c|c|c|}
\hline Variáveis & factor & $\begin{array}{c}\text { Valor } \\
\text { de v }^{1}\end{array}$ & $\begin{array}{c}\text { Valor } \\
\text { de }^{3}\end{array}$ & $\mathrm{R}^{\left.2{ }^{*}\right)}$ \\
\hline V1(item1) & SSA-am & .641 & .768 & .410 \\
\hline V6 (item6) & SSA-am & .672 & .740 & .452 \\
\hline V10 (item 10) & SSA-am & .752 & .659 & .566 \\
\hline V13 (item 13) & SSa-am & .762 & .647 & .581 \\
\hline V18 (item 18) & SSA-am & .694 & .720 & .482 \\
\hline V20 (item 20) & SSA-am & .751 & .661 & .563 \\
\hline V2(item 2) & SSA-prof & .672 & .741 & .451 \\
\hline V4 (item 4) & SSA-prof & .710 & .705 & .504 \\
\hline V9 (item 9) & SSA-prof & .613 & .790 & .376 \\
\hline V11 (item 11) & SSA-prof & .728 & .685 & .531 \\
\hline V16 (item 16) & SSA-prof & .680 & .733 & .462 \\
\hline V3 (item 3) & SSA-fam & .743 & .670 & .552 \\
\hline V5 (item 5) & SSA-fam & .762 & .647 & .582 \\
\hline V7 (item 7) & SSA-fam & .588 & .809 & .347 \\
\hline V12 (item 12) & SSA-fam & .759 & .652 & .576 \\
\hline V15 (item 15) & SSA-fam & .703 & .711 & .494 \\
\hline V19 (item 19) & SSA-fam & .773 & .635 & .597 \\
\hline SSA-am & Fator 1 & .823 & .568 & $.677^{4}$ \\
\hline SSA- Prof & Fator 2 & .712 & .703 & $.506{ }^{4}$ \\
\hline SSA-fam & Fator 3 & .663 & .748 & $.440^{4}$ \\
\hline
\end{tabular}

Legenda: SSA-am = percepção de apoio social dos amigos; SSA-prof = percepção de apoio social dos professores; SSA-família= percepção de apoio social da família;

${ }^{1}$ loading do fator em cada variável observada; ${ }^{3}$ variância residual do item(variência-erro);

(*)Variância do fator explicada por cada item/variável observada.

${ }^{4}$ Variância do erro associado às variáveis (fatores) latentes (“disturbance").

Nota: todos os valores apresentados se encontram padronizados ou estandardizados.

\section{Discussão dos resultados}

A assumpção de que um questionário ou escala destinado a avaliar uma característica não diretamente observável no sujeito, é um instrumento que traduz de forma fidedigna e válida essa característica, não deve ser tomada de ânimo leve. Um questionário é, apenas, um mapa da realidade que se pretende observar (Maia, 1996). Contudo, continuando a metáfora, se se quer avançar na investigação e intervenção psicológica, é desejável que os mapas utilizados orientem de forma correta. Para tal avaliam-se 


\section{Cristina Antunes}

algumas qualidades psicométricas da escala SSA, nomeadamente sua consistência interna e validade fatorial.

Como verificado nos estudos de consistência interna da escala SSA, os valores apresentados eram mais baixos nos adolescentes do $7^{\circ}$ e $8^{\circ}$ anos de escolaridade, em particular nos rapazes. Se não há dúvida que os itens da escala espelham todos a característica avaliada, é possível que, nestes casos, a escala também reflita dificuldades na resposta aos itens, devido à fase de desenvolvimento cognitivo em que os adolescentes se encontram. Quanto mais dificuldades tiverem, maior a variância-erro associada a cada item e mais baixa a consistência das respostas. É este tipo de consideração que levou a retirar os itens com formulação negativa. A vantagem potencial destes itens, no sentido de quebrar um eventual enviesamento nas respostas dos sujeitos, não compensa a desvantagem, nestas idades. A heterogeneidade excessiva introduzida nas respostas só pode ser explicada pela dificuldade dos alunos em lidar com o significado semântico da dupla negação. Constatase que a estrutura fatorial da escala é semelhante nos alunos mais novos ( $7^{\circ}$ e $8^{\circ}$ ano) e nos mais velhos ( $9^{\circ}$ ao $11^{\circ}$ ano) se não forem considerados todos com formulação negativa.

Para testar a multidimensionalidade da escala SSA, recorreu-se à técnica de modelação de estruturas covariantes. A escala SSA revelou, neste estudo, possuir uma estrutura fatorial hierárquica, com fatores de primeira e segunda ordem e itens a avaliar, com a mesma magnitude, o respetivo fator, embora seja diferente a variância-erro associada a cada item. $\mathrm{O}$ fato de os itens espelharem os fatores com igual magnitude é, sem dúvuida, um dado significativo, pela assumpção da homogeneidade das sub-escalas; levanta, contudo, a questão da eventual redundância dos itens: pergunta-se se, eventualmente, a escala não avaliaria com precisão idêntica a percepção de apoio social emocional dos três grupos considerados, reduzindo o número de itens por dimensão.

É digno de registo o fato dos resultados traduzirem a importância crescente que o grupo dos amigos e o apoio social que deles assume no início da adolescência. De fato, verificou-se que o fator percepção de apoio social dos amigos é o que explica maior variância da percepção de apoio social em ge- ral, na sub-amostra do $7^{\circ}$ e $8^{\circ}$ anos, comparativamente à do $9^{\circ}$ ao $11^{\circ}$ anos.

É a hoje consensual que o recurso à técnica de modelação de estruturas covariantes para avaliar a multidimensionalidade duma escala, pela sua exigência, transmite alguma segurança no estudo das qualidades psicométricas das de avaliação de constructos psicológicos. No entanto, não é legítimo concluir que os modelos considerados satisfatórios numa determinada amostra sejam generalizáveis a todas da mesma população ou de outras. Para isso, há necessidade de se proceder ao estudo da invariância fatorial dos modelos, ou seja, replicá-los noutras amostras da mesma ou de outra população e observar a sua adequação. A partir dos resultados deste estudo pode-se afirmar que a escala SSA parece ter fidedignidade e validade fatorial que a tornam apropriada para a utilização com adolescentes que frequentam o terceiro ciclo do ensino básico e o secundário.

\section{Referências Bibliográficas}

Almeida, J.P.,Reis, I.L. \& Norton, L. (1999). Apoio psicossocial, auto-conceito e adaptação psicológica de crianças sobreviventes de leucemia. Em A. P. Soares, S. Araújo \& S. Caires, Avaliação psicológica: Formas contextos (v.VI, pp. 6572). Braga: APPORT.

Antunes, C. (1994). O apoio social e o conceito de si próprio na adolescência. Dissertação de Mestrado. FPCE, Universidade do Porto, Porto.

Antunes, C. \& Fontaine, A. M. (1995). Diferenças na percepção de apoio social na adolescência: Adaptação do "social support appraisals". Cadernos de Consulta Psicológica, 10/11, 115127.

Antunes, C., \& Fontaine, A. M. (1996). Relação entre o conceito de si próprio e percepção de apoio social na adolescência. Cadernos de Consulta Psicológica, 12, 81-92.

Antunes, C., \& Fontaine, A. M. (2000). Relations between self-concept and social support appraisals during adolescence: A longitudinal study. Psychology: The Journal of the Hellenic Psychological Society, 7, 339-353. 
Arbuckle, J. L. (1997). Amos Users' Guide: Version 3.6. Chicago: SmallWaters Corporation.

Bentler, P. M. (1990). Comparative fit indexes in structural models. Psychological Bulletin, 107, 238-246.

Bentler, P. M. (1995). EQS: Structural Equations Program Manual. Encino, CA: Multivariate Software Inc.

Bentler, P. M., \& Weeks, D. G. (1980). Linear structural equation with latent variables. Psychometrika, 45, 289-308.

Browne, M. W. \& Cudeck, R. (1993). Alternative ways of assessing model fit. Em K. A. Bollen \& J. S. Long (orgs.), Testing structural equation models (pp.136-162). Newbury Park, C.A.: Sage.

Cobb. S. (1976). Social support as a moderator of life stress. Psychosomatic Medicine, 38, 300-314.

Colarossi, L. G., \& Eccles, S. (2003). Differential effects of support providers on adolescents' mental health. Social Work Research, 27 (1), 19-30.

DuBois, D. L., Burk-Braxton, C., Swenson, L. P., Tevendale, H. D., Lockerd, E. M., \& Moran, B. L. (2002). Getting by with a little help from self and others: Self-esteem and social support as resources during early adolescence. Developmental Psychology, 38 (5), 822-839.

Epstein, J. L. (1989). The selection of friends: Changes across the grades and in different school environments. Em J. Berndt \& G. Ladd (Orgs.), Peer relationships in child development (pp. 158-187). New York: Wiley.

Faria, M. C. C. S. (1999). Escala de apoio social instrumental e expressivo - IESSS. Em A. P. Soares, S. Araújo \& S. Caíres (Orgs.), Avaliação psicológica: Formas e contextos (Vol. VI, pp. 125-135). Braga: APPORT.

Furman, W., \& Burmester, D. (1985). Children's perceptions of the personal relationships in their social network. Developmental Psychology, 21, 1016-1024.

Giles, D. C. (2002). Advanced research methods in psychology. New York: Routledge.
Percepção de apóio social na adolescência 365

Harter, S., Waters, P., \& Whitesell, N. R. (1998). Relational self-worth: Differences in perceived worth as a person across interpersonal contexts among adolescents. Child Development, 69, 756766.

Heller, K., Swindle, R., \& Dusenbury, L. (1986). Component social support processes: Comments and integration. Journal of Consulting and Clinical Psychology, 54, 466-70.

Helsen, M., Vollebergh, W., \& Meeus, W. (2000). Social support from parents and friends and emotional problems in adolescence. Journal of Youth and Adolescence, 29 (3), 319-335.

Jöreskog, K. G., \& Sörbon, D. (1983). Lisrel VI: User's reference Guide. Mooresville, Em Scientific Software.

Larose, S., \& Bernier, A. (2001). Social support processes: mediators of attachment state of mind and adjustment in late adolescence. Attachment \& Human Development, 3 (1), 96-120.

MacDonald, R. P., \& Marsh, H. W. (1990). Choosing a multivariate model: Noncentrality and goodness of fit. Psychological Bulletin, 107, 238-246.

Maia, J. A. R. (1996). Um discurso metodológico em torno da validade de constructo: Posições de um Lisrelita. Em L. S. Almeida, S. Araújo, M. S. Gonçalves, C. Machado \& M. R. Simões (Orgs.), Avaliação Psicológica: Formas e contextos (Vol. IV, pp. 43-50). Braga: APPORT.

Marsh, H. W., \& Hocevar, D. (1985). Applications of confirmatory factor análisis to the study of selfconcept: First and higher order factor models and their invariance across groups. Psychological Bulletin, 97, 562-582.

Meeus, W. (1999). Parental and peer support, identity development and psychological wellbeing in adolescence. Comunicação apresentada na IX Conferência Europeia de Psicologia do Desenvolvimento, Spetses, Grécia.

Meeus, W., Helsen, M., \& Vollebergh, W. (1996). Parents and peers in adolescence: From conflict to connectedness. Em L. Verhofstad-Deneve, J. Kenhorst \& C. Braet (Orgs.), Conflict and 
development in adolescence (pp. 103-115). Leiden: Dswo Press.

Peixoto, F. J. (1999). Escala de percepção da relação com a família. Em A. P. Soares, S. Araújo \& S. Caíres (Orgs.), Avaliação Psicológica: Formas e contextos (Vol. VI, pp. 468-474). Braga: APPORT.

Pekrun, R. (1990). Social support, achievement evaluations and self-concepts in adolescence. Em L. Oppenheimer (Org.), The self-concept: European perspectives on its development, aspects, and applications (pp. 107-119). Berlin: Springer.

Pinheiro, M. R. M., \& Ferreira, J. A. G. (2000). O questionário de suporte social: Adaptação e validação da versão portuguesa do Social Support Questionnaire (SSQ6). Psychologica, 30, 315-333.

Sarason, I. G., Sarason, B. R., Shearin, E. N., \& Pierce, A. R. (1987). A brief measure of social support: Practical and theoretical implications. Journal of Social and Personal Relationships, 4, 497-510.

Scholte, R. H. J., van Lieshout, C. F. M., \& van Aken, M. A. G. (2001). Perceived relational support in adolescence: Dimensions, configurations, and adolescent adjustment. Journal of Research on Adolescence, 11 (1), 71-94.

Schumacker, R. E., \& Lomax, R. G. (1996). A beginner's guide to structural equation modeling. Mahwah, New York: LEA.

Sprinthal, N., \& Collins, W.A. (1988). Adolescent psychology: A developmental view. New York: Random House.

Vaux, A. (1988). Social support: Theory, research and interventions. New York: Praeger.

Vaux, A. (1992). Assessment of social support. Em H. O. F. Veiel \& U. Baumann (Orgs.), The meaning and measurement of social support (pp. 193-216). New York: Hemisphere.

Vaux, A., \& Harrison, D. (1985). Support network characteristics associated with support satisfaction and perceived support. American
Journal of Community Psychology, 13, 245269.

Vaux, A., Philips, J., Holly, L., Thompson, B., Williams, D., \& Stewart, D. (1986). The social support appraisals (SSA) scale: studies of reliability and validity. American Journal of Community Psychology, 14, 195-220.

Veiga, F. V. (1999). Os direitos dos jovens na família. Em A. P. Soares, S. Araújo \& S. Caíres (Orgs.), Avaliação Psicológica: Formas e contextos (Vol. VI, pp. 604-615). Braga: APPORT.

Wentzel, K. R. (1998). Social support and adjustment in middle school: The role of parents, teachers and peers. Journal of Educational Psychology, 90, 202-209.

Youniss, J., \& Smollar, J. (1989). Adolescents' interpersonal relationships in social context. Em J. Berndt \& G. Ladd (Orgs.), Peer relationships in child development (pp. 300-316). New York: Wiley.

Ystgaard, M., Tambs, K., \& Dalgard, O. S. (1999). Life stress, social support and psychological distress in late adolescence: A longitudinal study. Social Psychiatry and Psychiatric Epidemiology, 34, 1219. 